Article

\title{
Four Different Multimodal Setups for Non-Aerial Vehicle Simulations-A Case Study with a Speedboat Simulator
}

\author{
Sergio Casas *, Marcos Fernández and José V. Riera \\ Institute of Robotics and Information and Communication Technologies (IRTIC), Universitat de València, \\ Valencia 46980, Spain; Marcos.Fernandez@uv.es (M.F.); J.Vicente.Riera@uv.es (J.V.R.) \\ * Correspondence: Sergio.Casas@uv.es; Tel.: +34-963-543-557 \\ Academic Editor: Adrian David Cheok \\ Received: 29 March 2017; Accepted: 17 May 2017; Published: 19 May 2017
}

\begin{abstract}
Vehicle simulators are multimodal interactive applications used in many human activities with different purposes. However, they are sometimes expensive systems that need to be carefully studied before they are designed and built, since some simulators can actually be much more expensive than the simulated vehicle. This is an important issue, although it is sometimes overlooked in scientific research. This paper proposes four different setups (with a variety of visual, sound, motion generation, and user-input interfaces) for non-aerial vehicle simulation, using a speedboat simulator as a case study. These setups are analysed in terms of their cost and their effectiveness is discussed. Rough figures are provided to give a comparative insight into the economic order of magnitude necessary to design and build a vehicle simulator.
\end{abstract}

Keywords: vehicle simulation; cost; multimodal; interaction; virtual reality; speedboat

\section{Introduction and Related Work}

The history of modern vehicle simulation dates back to the early years of the twentieth century [1]. There are records of primitive flight simulators as early as 1910 [2]. Computer-based vehicle simulations appeared after World War II, although the earlier visual systems were based on TV cameras with mock-ups or pre-recorded scenarios. Synthetic images appeared in the 1970s and 1980s. Yet, there were not realistic visual systems almost until the end of the century. The use of motion platforms to add self-motion perceptual cues and achieve physical or perceptual validity [3] in vehicle simulators is not new either. They were first applied to simulations in the 1950s, although earlier devices, like the Link Trainer, tried to include this effect in simulators, with little success [4]. During the 1960s and especially the 1970s, when Stewart-Gough [5] parallel manipulators were introduced and applied for vehicle simulation, these kinds of systems became common in flight simulators [6].

Vehicle simulators are used in many knowledge areas with different objectives. They can be used for skills training, so that pilots learn how to control a vehicle and practice these kind of skills. They can be used to train and learn protocols (safety, rescue, landing, take off, refuelling, etc.) when pilots already know how to control the vehicle, but need further training in high level strategies. They are also used for scientific research to study the behaviour of particular groups of pilots. They can be used for entertainment, education, edutainment, safety awareness, etc. Different uses and objectives create different necessities and requirements, which significantly modify the cost-effectiveness ratio of the vehicle simulator. Therefore, it is essential to design the simulator according to its objectives, so that the cost-effectiveness ratio is minimized. In this regard, it is important first to know the different interaction paradigms and multimodal perceptual cues that can be added to the simulator. 
Vehicle simulation is considered of the utmost importance and the vehicle simulation industry is probably the leading industry in the simulation field, as vehicle simulators provide many benefits for companies, vehicle operators (pilots, drivers), and for society as a whole (throughout this work, we use the word pilot, referring to vehicle operators, regardless of the vehicle type). Vehicle simulators provide $[1,7,8]$ :

- An increase in safety and a reduction of risks.

- Cost reduction, since failures can be detected in early phases.

- Greater trial availability, since the simulator, unlike some vehicles, is usually always available.

- An increase in the availability of trainers and students, since the simulator can be installed in many different places, unlike real vehicles, which may be confined to a port or circuit.

- The possibility of simulating accidents without causing damage to the pilots.

- The possibility of recreating a variety of situations and weather conditions without having to wait until they happen (if they do).

- The possibility of repeating the same tests under the same conditions.

- The ability to evaluate tests objectively and perform debriefings with pilots.

- Transfer of skills and training.

A great deal of vehicle simulators, with different setups, for many different vehicle types have been proposed: planes [9-12], helicopters [13-15], cars [16-18], motorcycles [19-21], trucks [22], tractors [23], sail ships [24], engine-based ships/boats [25-28], bicycles [29,30], or even tricycles [31]. Some of them include multimodal perceptual cues and realistic interaction systems. The spectrum of setups is very wide, and the complexity of the simulators has generally increased over the years.

Planes are expensive and complex vehicles and the need for expert pilots is fundamental since accidents with aircrafts are, more often than not, fatal. Therefore, flight simulators have led the research in vehicle simulation ever since computers started to be applied to this field. The application of simulation techniques to other types of vehicles has led to many research areas, where simulation is applied with different interaction paradigms and multimodal perceptual cues. In this regard, a flight simulation is obviously different from driving or ship simulations. Physics, vehicle controls, cockpit complexity, training necessities, pilot behaviour, and expectances are different, although a great deal of common ground can be established and some of the simulation software could be shared, producing synergies. However, the economic side has always been an issue, and some vehicles are less expensive than their corresponding vehicle simulators. A vehicle simulator is designed to serve a purpose, and therefore, it may be sometimes hard to justify building a simulator that is much more expensive than the actual vehicle, unless safety reasons compel its development. This is one of the reasons for the large use of flight simulators with respect to other areas. This does not mean that the simulation of other types of vehicles is not as important as a flight simulation. On the contrary, a driving simulator to prevent road accidents, for instance, can be also very cost effective.

The analysis of the effectiveness of simulators constitutes a vibrant, interesting, and very important research field, since the variety of simulator types and goals creates different needs, which, in turn, implies different assessment methods. In some simulators, it is sufficient to analyse the behavioural validity (the extent to which the simulator replicates the real behaviour of the vehicle) [32]. For training simulators, the effectiveness of the simulation is usually determined by the amount of training time in the real vehicle that can be saved by using the simulator [33]. Simulation systems can be assessed subjectively (gathering opinions about how they are perceived by their users), although objective evaluation is usually preferred, except for the assessment of motion fidelity, which is a controversial topic with different opinions [6,34]. Simulation effectiveness can also be evaluated directly by measuring the goals of the simulator (if possible), or indirectly, by measuring variables that are thought to be related to the purpose of the simulator [35]. In any case, the assessment of the effectiveness of a simulator depends on its purpose, and few general rules can be provided. 
Since the complexity of many of these simulation systems usually make them very expensive, in recent years, there has been a renewed interest in low-cost vehicle simulators [10,36,37], so that effective but inexpensive vehicle simulation systems can be designed and implemented. Unfortunately, the analysis of the cost of these systems is something rarely done in the academic community and it is usually left for the simulation industry.

For this reason, this paper presents four different setups for vehicle simulations, analysing their costs, while discussing their possible effectiveness. The paper focuses on non-aircraft simulation, since it is a less researched field. We use a speedboat simulator as a case study, to illustrate the analysis and provide actual cost figures.

\section{Materials and Methods}

\subsection{Components of a Vehicle Simulation}

A vehicle simulator is composed of several software modules and hardware devices that work together to perform the simulation. These modules and devices can be grouped into the following subsystems:

- Physics system. It is the core of the simulation. It simulates the physical behaviour of the vehicle by means of a suitable model of the simulated vehicle, which is called the virtual vehicle.

- Task system. It is usually a software module in charge of guiding the execution of the simulation so that the vehicle simulator serves its purpose and objectives. In a training simulator, for instance, it would be in charge of providing the conditions of the training session and/or supervising the execution of the exercises. It is highly dependent on the simulator type and objectives, although it can be considered an integral part of the vehicle simulator, since it controls several key aspects of the simulation.

- Visual system. It provides visual cues for the pilot.

- Sound system. It is used to generate acoustic perceptual cues.

- Motion system. It generates real motion for the pilot, in order to provide self-motion perceptual cues that are consistent with the movements of the virtual vehicle.

- User-input system. It includes the reproduction of the vehicle controls (although they are sometimes simplified). It serves as the entry point of user's actions to the simulator.

- User-output system. It includes the gauges or indicators of the vehicle (revolution counter, speedometer, fuel indicator, etc.), which can be virtually or actually reproduced. Together with the previous system, they correspond with the cockpit of the vehicle.

Figure 1 summarizes the different elements of a vehicle simulator. Elements in blue represent hardware devices. Arrows represent the communication of information.

The task module and the physics module control the simulation. The former sets and supervises the conditions for the vehicle simulation, while the latter controls almost all the aspects of the behaviour of the virtual vehicle. Modern vehicle simulators require that this module calculates the dynamic equations that model the physical behaviour of the vehicle. This vehicle model is controlled by means of sensorized vehicle controls that are read through the user-input module.

Audio-visual cues are managed by visualization and sound software modules, which render audio-visual cues through the visualization and sound hardware. If a more realistic simulation is considered, some of these visual cues can come in the form of actual vehicle gauges, which are controlled and displayed via the user-output module. In some cases, they can be (virtually) integrated within the visual module. 


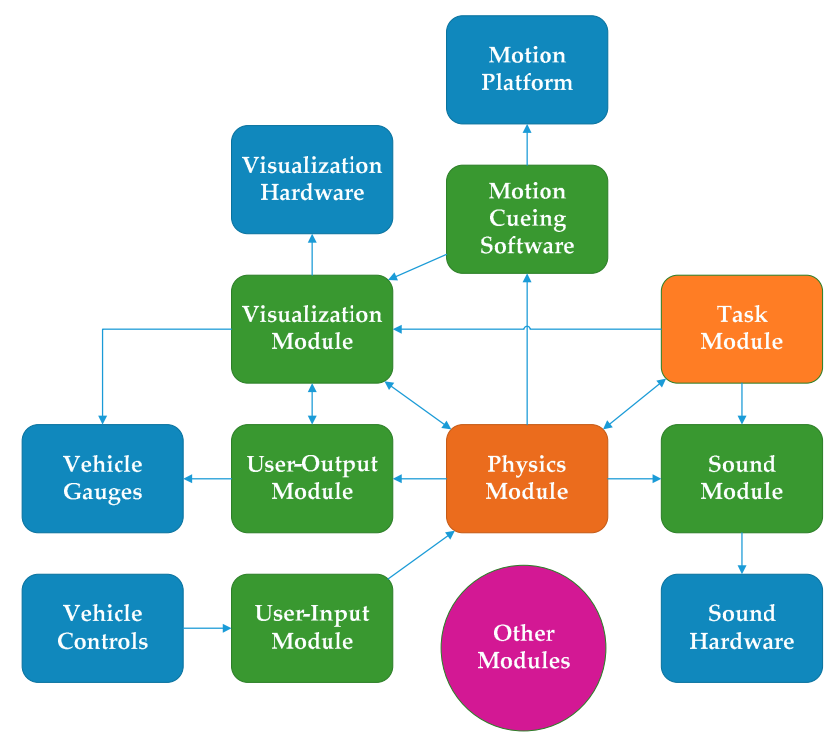

Figure 1. Scheme of the components of a vehicle simulator.

Finally, self-motion perceptual cues are generated by moving the pilot by means of a motion platform. A Motion Cueing Algorithm (MCA) [38], in combination with software for the motion platform actuators, is responsible for this task. It is required that visual and inertial cues be rendered in a synchronized way. In fact, some designs place the visual system on top of the motion platform. This causes the motion hardware to slow down its movements when the visual system is too heavy. Thus, the visual system is often left on the ground and the motion cueing software performs corrections to account for the discrepancies between the expected perspective, and the current one.

Other modules could be added to include Artificial Intelligence or provide other perceptual multimodal cues, such as tactile, haptic, olfactory, thermal, pressure, etc. In this regard, it is important to clarify that the proposed structure represents one possible general approach for organizing a vehicle simulator. This could be adapted to fulfil the particular requirements of the simulator, and other approaches can be proposed where some components may be absent or some others may be needed.

\subsection{Case Study: A Speedboat Simulator}

A speedboat or motorboat is a boat propelled by an engine, which is designed to achieve high speeds in a water medium. They are used for recreational activities, and rescue and surveillance tasks, among others. Although speedboats can significantly vary in size, configuration, and power, the most common setup is between four and eight meters long with an engine in the back. Most of them are controlled by a rudder, similar to a car steering wheel. The propeller is usually controlled by means of an analogic throttle lever. Some of them incorporate a compass, radio communications, and visual aids for the pilot, such as fuel or revolution counters.

The motion of a speedboat is highly dependent on sea conditions. In any case, they tend to pitch up with throttle, pitch down abruptly when the throttle is cut, and roll from side to side if sea waves are high, especially if no throttle is applied [39]. They have no brakes, since the high friction of water makes them unnecessary. Most of them have no windshield or pilot protection structure either, and therefore, the sound of the engine and the wind itself are quite noticeable. They are designed to achieve high speeds, but due to the nature of water, they do not usually reach more than 20-30 knots (less than $60 \mathrm{~km} / \mathrm{h}$ ) at full throttle. Acceleration and tilt are quite noticeable, though, because the vehicle is constantly moving, either by the action of the propeller or by the sea waves.

The simulation of this kind of vehicle (see Figure 2) is rare, as most of the works dedicated to marine vehicle simulation are focused on large ships, which are much more expensive. However, this vehicle represents an interesting case study of interactive multimodal setups, because the absence of a 
windshield makes extra-visual cues (wind, sound, etc.) important, it exhibits a great motion range, and its controls are neither too simple nor as complex as those of a plane.

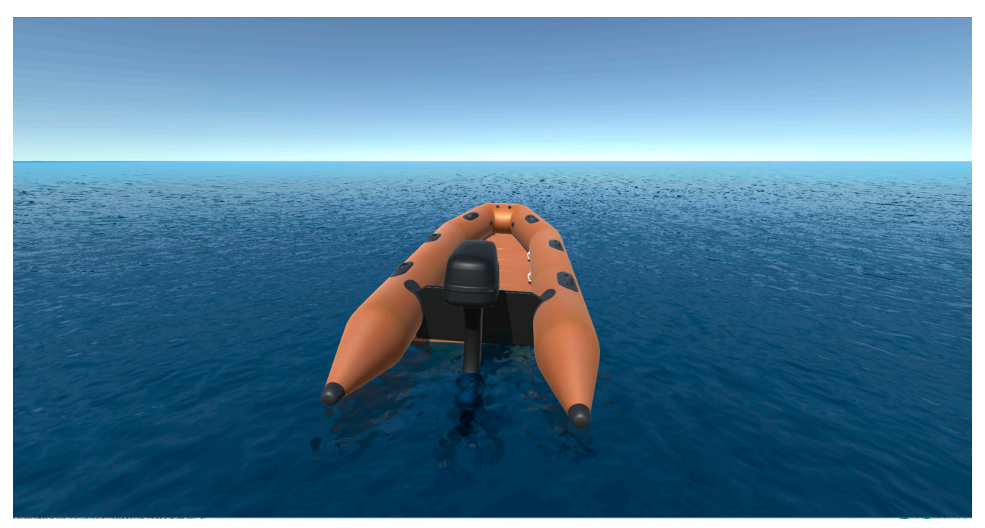

Figure 2. Snapshot of the visual module of a speedboat simulator.

In addition, the need for training people in using this kind of vehicle is important, and the risks associated with accidents using a speedboat represent an opportunity to use vehicle simulators. In fact, this kind of simulator could be of interest for many reasons. First, to help trainees learn how to control this vehicle - there are only a few controls, but sea conditions (waves, currents) are constantly varying, making this task quite complex. Second, to study the human factors and the reaction of pilots to different sea/weather conditions. Third, to train safety and rescue protocols (this kind of vehicle is extensively used in sea rescue patrols). Fourth, to improve these protocols by analysing possible flaws and risks in their specification and design.

\subsection{Setup \#1}

In the following sections, we propose several setups for vehicle simulators, using the speedboat simulator as a case study. One of the simplest setups for a vehicle simulator is to use a monitor and a USB game steering wheel (or even a joystick). Of course, not all vehicles are controlled by a steering wheel, but most of the non-aerial vehicles (cars, trucks, tractors, buses, ships, boats, etc.) are or may be. Aircrafts have complex control systems and require some sort of cockpit reproduction, but might be adapted to this setup if the vehicle simulation is intended exclusively for entertainment. The number of simulation-oriented steering wheels available in the market is significant and even seats and simple cockpits can be found at reasonable prices. Monitors are also cheap and can be substituted by a TV screen to produce a larger visualization system. Although most TV screens provide decent sound generation, a dedicated sound system can be added, so that the speakers can be placed in different locations.

For the speedboat simulator case, the proposed setup \#1 is composed of:

- A computer. This is needed to run the physics simulation and generate the audio-visual content.

- $A 50^{\prime \prime} T V$ screen. This represents the hardware of the visual system.

- A 2.1 speaker system, to generate simple but effective spatial sound.

- A USB racing steering wheel. Although, these devices are usually employed for driving simulation, they can serve as the speedboat control system.

\subsection{Setup \#2}

In order to enrich the experience of the users, this setup proposes a much more complex design. To upsize the display system, a reasonable solution is to use a big white screen and a projector. This creates a much more immersive visualization that contributes to enhancing the user experience. Obviously, 
the larger the screen, the more expensive the solution is. In addition, if the screen is too large, more than one projector could be needed to fill the surface.

An evident improvement over the previous setup is to include the actual vehicle controls in the simulator. As a complete vehicle or even a cockpit are usually very expensive, a reasonable solution is to recreate only the controls and gauges of the vehicle. This is highly dependent on the particular vehicle being simulated. Nevertheless, in all cases, the controls need to be sensorized so that the actions on the vehicle controls are communicated to the computer running the simulator software. This is usually accomplished by placing sensors, like potentiometers or tilt-sensors, in the vehicle controls. Unlike controls, gauges can be virtually reproduced.

Another way to enhance the user experience is to add self-motion generation, so that users perceive motion in a way that is similar to a real situation. This is usually accomplished by placing the user and the vehicle controls over a motion platform. This device would generate motion cues consistent with the visual information. Thus, it needs to be properly synchronized with the visual system and receives data from the physics module. The complexity and the utility of these devices depend on the number of degrees of freedom (DOF), the size, and the power of the actuators. Many studies show that the addition of motion cues improves the perceptual experience or control behaviour of the pilots $[12,40,41]$. However, it is also true that bad motion cueing could sometimes be even worse than no motion. Therefore, it is very important to study the motion capabilities of the motion platform so that it is the right one for the simulated vehicle. A proper characterization of the vehicle motion and the perceptual cues associated with it should be performed before choosing/building the motion mechanism, as explained in [39].

For the speedboat simulator case, the proposed setup \#2 is composed of:

- A computer. It should not be very different from the one in setup \#1.

- Two projectors and a $2 \times 3 \mathrm{~m}$ (width $\times$ height) white flat reflective surface. It substitutes the TV screen of setup \#1.

- A 2.1 speaker system, to generate spatial sound.

- A 2-DOF pitch-roll motion platform (similar to the one shown in Figure 3). This is probably the simplest motion system design, yet it provides clues about the pitch-roll motion of the speedboat, which are the most important ones for this vehicle [39].

- A real speedboat control post with rudder and throttle (as seen in Figure 4). These controls are sensorized by means of potentiometers. Although the sensors are usually cheap, the design work to fit them into the real devices can be complex.

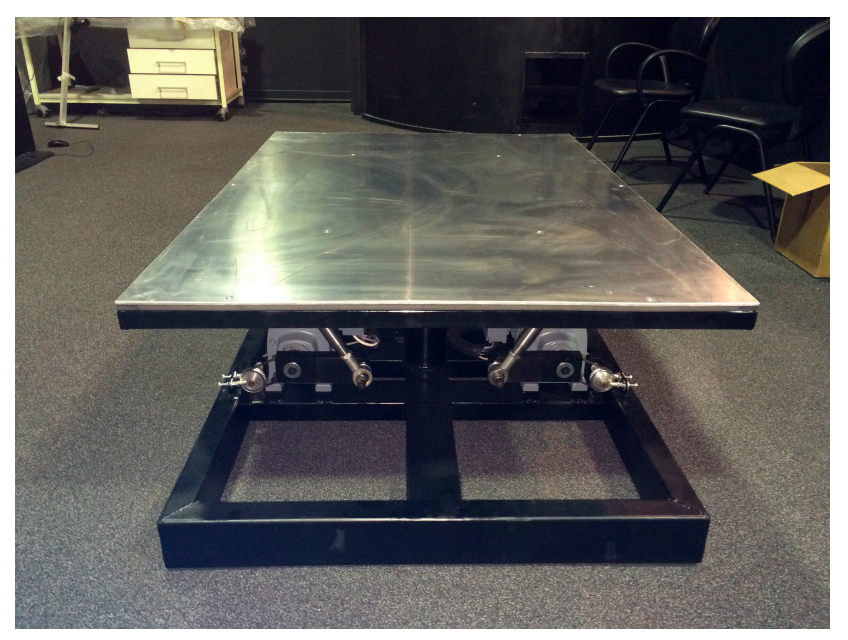

Figure 3. Example of a 2-DOF pitch-roll motion platform. 


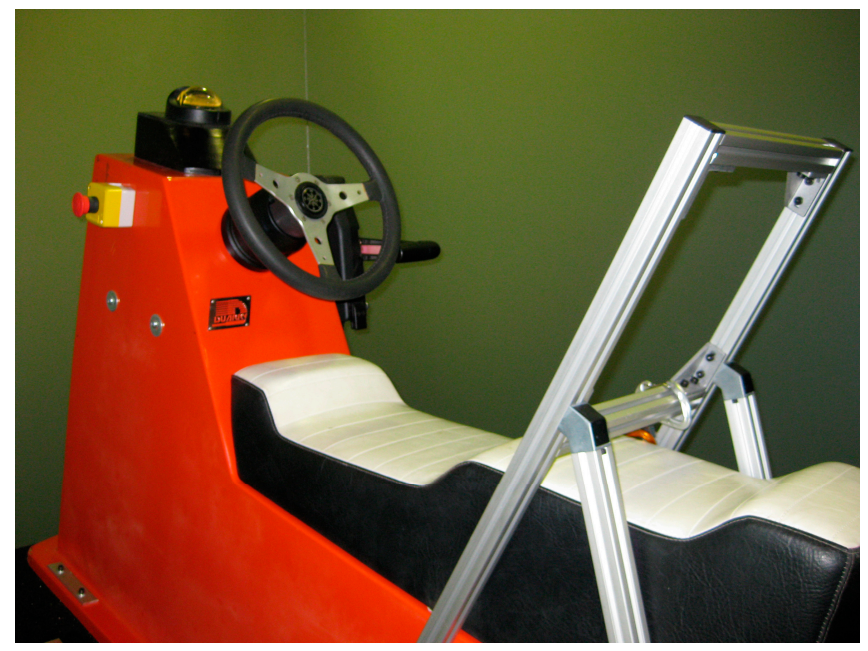

Figure 4. Example of vehicle controls for the speedboat simulator.

\subsection{Setup \#3}

More improvements can be proposed for the vehicle simulator. Regarding the visual system, a single flat screen still represents visual information differently from a real world situation, since the human eye has a large field of view (FOV) and extra-foveal information contributes to the immersion sensation. Therefore, it is advised that the simulator visual system covers at least $180^{\circ}$ of horizontal FOV with respect to the pilot position.

The motion system can also be enhanced by adding capabilities to the motion platform. A natural improvement is to include additional DOF.

With the vehicle controls already sensorized, improvements can be made by adding actual gauges of the vehicle, instead of showing them virtually. In the case of the speedboat, the most important one is the navigation compass, which can be actuated with a small motor to move the north mark consistently with the heading of the simulated vehicle. Another important improvement in this case study is to generate wind with an electrical fan controlled by the simulator, so that the amount of generated wind is directly proportional to the speed of the simulated vehicle. This fan has to be synchronized with the physics module. Wind sensation is very important in vehicles with no windshield.

For the speedboat simulator case, the proposed setup \#3 (see Figure 5) consists of:

- A computer. It should not be very different from the one in setup \#2.

- Six projectors and three white flat reflective surfaces (two projectors per surface) with the same size as in setup \#2. The use of three screens allows the creation of different visual setups (U-shaped or trapezium-shaped) that are much more immersive than the one in setup \#1 (see Figure 6).

- A 5.1 speaker system, to generate spatial sound. The use of three screens allows placing the speakers in different locations, increasing the quality of the spatial sound.

- A 3-DOF heave-pitch-roll motion platform. This is still a simple motion system design, yet it provides the most important inertial clues of the speedboat (except for the yaw rotation, which is absent). The inclusion of heave motion allows the simulation of vertical acceleration caused by the boat hitting water.

- A real speedboat control post with an actual compass plus a small motor to move its north mark (see Figure 7).

- A wind generator plus a frequency inverter to control the speed/power of the fan. 


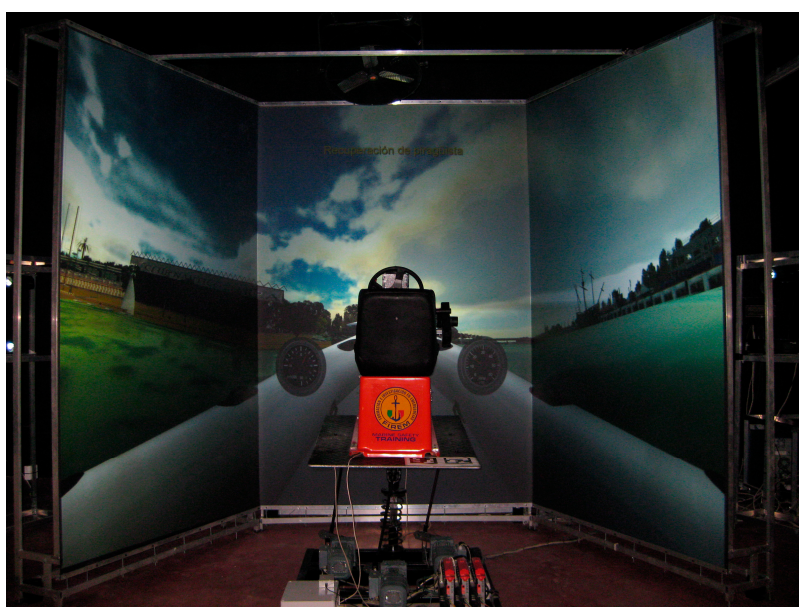

Figure 5. One possible realization of setup \#3.

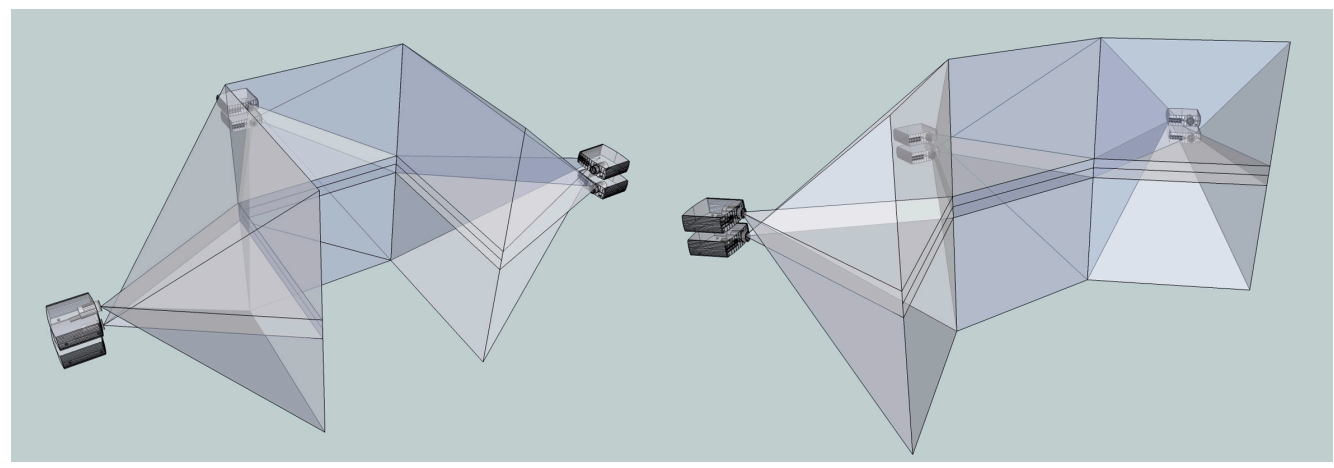

Figure 6. Two different display setups with three flat screens and six projectors.

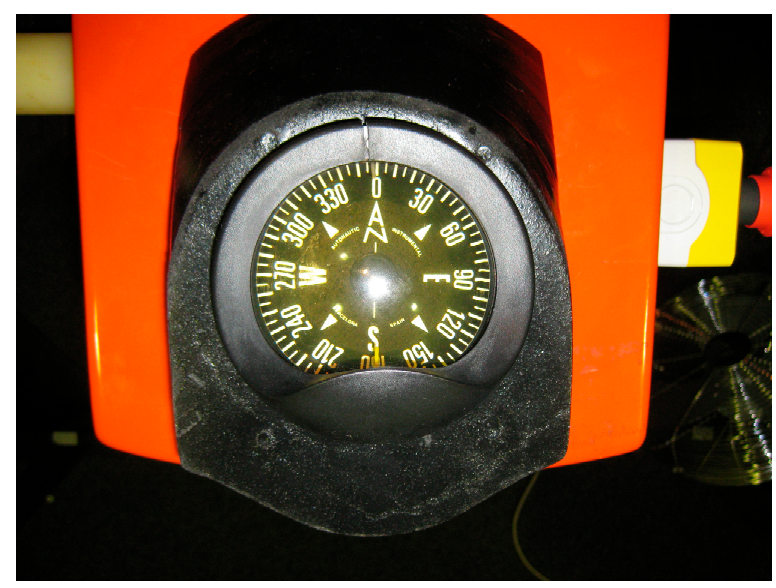

Figure 7. A motorized heading compass.

\subsection{Setup \#4}

The visual system in setup \#3 can be improved by using cylindrical screens instead of the flat ones. The problem of flat screens is that they show three different perspective projections that match in adjacent borders, but they represent a visual scenario that is slightly different from the one that the human eye experiences. With cylindrical surfaces, the projection can be made in a way much more similar to the one that the human eyes expects. However, these screens are harder to build, and, therefore, are more expensive. 
Self-motion can be enhanced by using a 6-DOF motion platform. The fact that it possesses six DOF does not mean, however, that it has unlimited motion capabilities. In fact, the translational displacement that motion platforms can offer are usually five or six orders of magnitude smaller than those of real vehicles are. The same does not hold true for rotational motion, which may be, in some cases, reproduced with a 1:1 scale. This leads to several problems for the design and tuning of the MCA. Improvements in the vehicle controls can only be made by including a real vehicle with sensorized controls, which is considerably expensive.

For the speedboat simulator case, the proposed setup \#4 consists of:

- A computer. It should not be very different from the one in setup \#3.

- Six projectors and three cylindrical reflective surfaces of similar sizes as the ones that are proposed in setup \#3.

- A 5.1 speaker system, to generate spatial sound.

- A 6-DOF Stewart-like motion platform, similar to the one shown in Figure 8. This represents a full-motion setup.

- A real speedboat. This represent the most realistic setup in terms of the cockpit (see Figure 9, where a real speedboat is used to build a simulator).

- A wind generator, as in setup \#3.

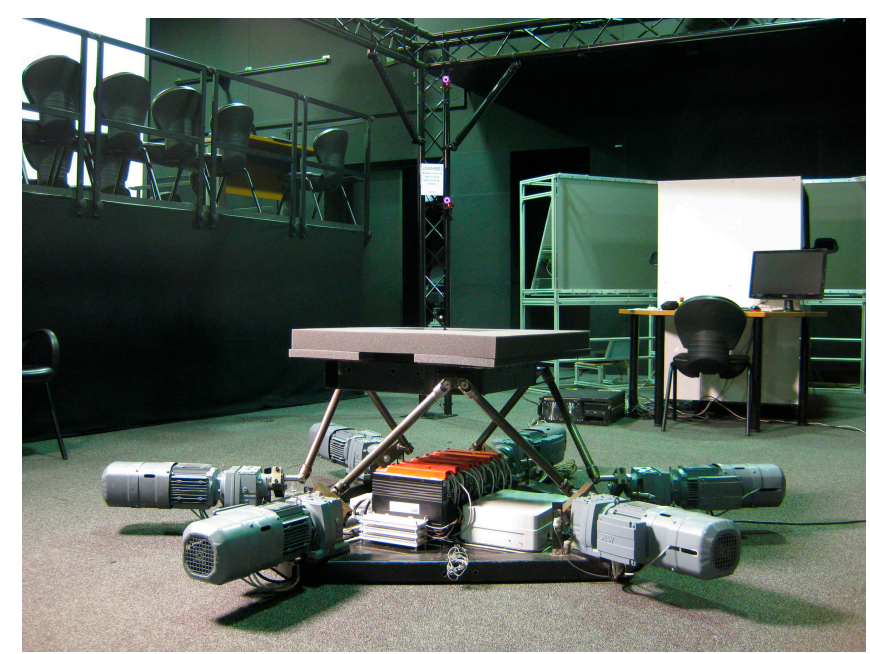

Figure 8. Example of a Stewart-like 6-DOF motion platform.

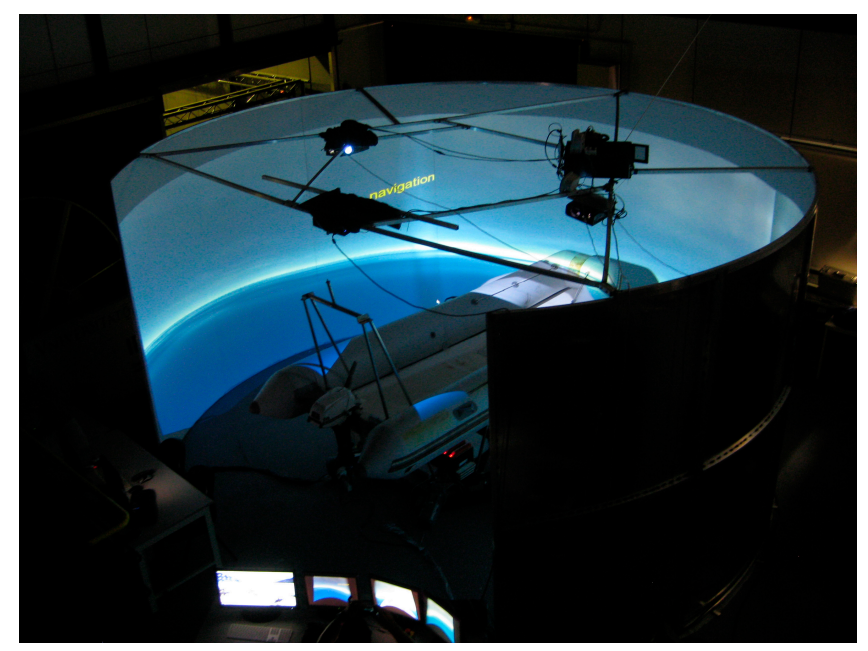

Figure 9. Full-boat vehicle simulation with cylindrical screens (vehicle controls are not shown). 


\section{Cost Analysis}

In this section, we present an analysis of the cost of building the four different setups. Rough figures are provided, since prices can change and depend on the country. Therefore, absolute values are not of the utmost importance. The important aspects are to study and compare the different solutions, identify differences in terms of the order of magnitude, break down and relate the different costs of the different setups, and be able to have a clearer picture of what it takes to choose between the different levels of interaction and multimodal perceptual cues.

As a general rule, we can establish that the cost of a vehicle simulation can be expressed by the following sum of terms:

$$
C=C_{H}+C_{S}+C_{V}+C_{A}+C_{C}+C_{M}+C_{E}
$$

where $C_{H}$ is the cost of the computer hardware to run the simulation, $C_{S}$ represents the cost of the software, $C_{V}$ is the cost of the visual system's hardware, $C_{A}$ represents the cost of the audio system's hardware, $C_{C}$ represents the cost of reproducing the vehicle controls, $C_{M}$ accounts for the cost of the motion system (if any), and $C_{E}$ represents some other possible extra costs.

Different setups have different costs, although some elements can be shared and assumed to remain constant. Let us denote the cost of each setup as $C_{i}$, with $i$ varying between one and four.

Using (1), the cost of setup \#1 can be approximated by:

$$
C_{1}=C_{C H W}+C_{S S W}+C_{T V}+C_{A 2.1}+C_{G I}
$$

where the terms in (1) have been broken down into the different actual elements of this setup: $C_{C H W}$ represents the cost of the computer hosting the software modules needed to run the simulation (physics, tasks, visualization, audio, etc.); $C_{S S W}$ represents the cost of these software modules; $C_{T V}$ is the cost of the $50^{\prime \prime} \mathrm{TV}$ screen; $C_{A 2.1}$ is the cost of a 2.1 sound system; and $C_{G I}$ represents the cost of a USB gaming steering wheel.

Similarly, the cost of setup \#2 can be approximated by:

$$
C_{2}=C_{C H W}+C_{S S W}+C_{M S W}+2 C_{P R}+C_{F S}+C_{B D C}+C_{A 2.1}+C_{P C}+C_{F M}+2 C_{D M}
$$

where $C_{M S W}$ represents the cost of the motion cueing software, $C_{P R}$ represents the cost of a projector, $C_{F S}$ is the cost of the white flat screen, $C_{B D C}$ is the cost of blending several images (additional hardware can be used although this is usually solved with dedicated software), $C_{P C}$ is the cost of acquiring and sensorizing the pilot's controls, $C_{F M}$ represents a fixed cost for building a motion platform, and $C_{D M}$ represents the cost of adding one DOF to the motion platform.

Changing the TV screen by two projectors should not affect $C_{C H W}$, so we can use the same computer hardware. Although the addition of motion cueing software would require some extra CPU power, we can assume that it is not necessary to change this computer because the one chosen for setup \#1 should already be a powerful one.

The cost of the simulation software $\left(C_{S S W}\right)$ is assumed to be equal to the case of setup \#1. Although some extra software effort should be made to acquire data from the vehicle controls, this should not be a great issue with respect to the similar process needed in setup \#1, and it can be neglected. On the contrary, the cost of the motion cueing software $\left(C_{M S W}\right)$ has been kept separate with respect to $C_{S S W}$, because it usually takes a great deal of effort and is not included in all vehicle simulators.

The term $\left[C_{F M}+2 C_{D M}\right]$ represents the cost of the 2-DOF motion generation hardware $\left(C_{M}\right)$. We assume that there is a fixed cost for designing and building a motion platform, and that this cost raises lineally with the number of DOF. Although this is an approximation, the most important costs of a motion platform are the actuators and their control hardware (servo-controllers or variable frequency drives). Usually, one actuator is required (plus its correspondent control hardware) per DOF. Thus, the assumption should be rather approximate. 
The cost of setup \#3 can be estimated by:

$$
C_{3}=C_{C H W}+C_{S S W}+C_{M S W}+3\left[2 C_{P R}+C_{F S}\right]+C_{B D C}+C_{A 5.1}+C_{P C}+C_{F M}+3 C_{D M}+C_{H C}+C_{W G}
$$

where $C_{A 5.1}$ accounts for the cost of a 5.1 speaker system, $C_{H C}$ accounts for the cost of a motorized heading compass, and $C_{W G}$ represents the cost of adding a wind generation system.

In this case, $C_{C H W}$ could be a little higher than in setup \#2, since the graphics card should allow the connection of six visual displays. Nevertheless, we assume that the original computer of setup \#1 can handle this without assigning possible extra costs to $C_{B D C}$. The cost of software can be maintained, since using a 3-DOF motion platform instead of a 2-DOF, and three views instead of one, requires only a small extra effort in terms of programming. Thus, neither $C_{S S W}$ nor $C_{M S W}$ need to be changed.

Regarding the visual system, we assume similar screens and projectors with respect to setup \#2. The same can be said for the vehicle controls and the fixed and relative costs of the motion platform $\left(C_{P C}, C_{F M}, C_{D M}\right)$.

Finally, the cost of setup \#4 can be estimated by:

$$
C_{4}=C_{C H W}+C_{S S W}+C_{M S W}+3\left[2 C_{P R}+C_{C S}\right]+C_{B D C}+C_{A 5.1}+C_{R B}+C_{F M}+6 C_{D M}+C_{H C}+C_{W G}
$$

where $C_{C S}$ represents the cost of a cylindrical white screen section (of a similar size with respect to the flat one) and $C_{R B}$ is the cost of acquiring a full boat and sensorizing its controls. The rest of the costs are assumed to remain as in setup \#3, although $C_{B D C}$ could be a little higher to include the distortion correction for projecting in a non-flat surface. To simplify, this is neglected in this analysis, since blending correction software usually includes warping and distortion correction.

Although many of these costs can vary, we can identify several critical points in this analysis. On the one hand, except for setup \#1, the quality and price of the visual system depends on the features of the projectors. This is represented by $C_{P R}$. On the other hand, the motion system's performance depends on the power of the actuators and the size of the motion platform's hardware pieces. This is represented by $C_{D M}$. Powerful motors are expensive and may require expensive and specialized control and hardware systems. For this reason, both figures $\left(C_{D M}\right.$ and $\left.C_{P R}\right)$ are estimated with ranges. The rest of the elements can be estimated with single figures, as seen in Table 1. Values are in Euro $(€)$ and are estimated for the Euro area.

Table 1. Setups' items and their approximate costs.

\begin{tabular}{ccc}
\hline Item & Description & Approximated Cost (€) \\
\hline$C_{C H W}$ & Computer hardware needed to run the simulation & 2000 \\
$C_{S S W}$ & Simulation software (except for motion cueing) & 25,000 \\
$C_{M S W}$ & Motion cueing software & 12,500 \\
$C_{T V}$ & $50^{\prime \prime}$ TV screen & 500 \\
$C_{P R}$ & Projector & {$[500-5000]$} \\
$C_{F S}$ & White flat screen $(2 \times 3 \mathrm{~m})$ & 1000 \\
$C_{C S}$ & White cylindrical screen section $(2 \times 3 \mathrm{~m})$ & 2000 \\
$C_{B D C}$ & Blend and distortion correction system & 500 \\
$C_{A 2.1}$ & 2.1 speaker sound system & 100 \\
$C_{A 5.1}$ & 5.1 speaker sound system & 300 \\
$C_{G I}$ & Steering wheel gaming interface & 300 \\
$C_{P C}$ & Sensorized pilot's controls & 2000 \\
$C_{R B}$ & Real boat sensorized controls & 6000 \\
$C_{F M}$ & Fixed cost for building a motion platform & 4000 \\
$C_{D M}$ & Cost per DOF in a motion platform & {$[2000-20,000]$} \\
$C_{H C}$ & Motorized heading compass & 500 \\
$C_{W G}$ & Wind generation system & 300 \\
\hline
\end{tabular}


For the cost of software, six months of effort are assumed necessary to build the simulation software, and three months to build the motion cueing software. Although the visualization of water and floating objects is complex, development tools like Unity make this task easier than some years ago. These figures represent the cost of a free navigation simulator, for skill learning, with no programmed tasks. Simulation software that needs more complexity could be much more expensive. Applying a salary of 50,000 $€$ per year to a software engineer, the costs of $C_{S S W}$ and $C_{M S W}$ can be estimated as $25,000 €$ and $12,500 €$. These figures are conservative since they assume that the simulator is not going to be licensed more than one time. Therefore, this cost can be significantly decreased if more than one instance of the simulator is built.

The total cost figures are shown in Tables 2-4 for setups \#2,\#3, and \#4, respectively. They are calculated applying Equations (3-5) with the values of Table 1. The cost of setup \#1 is calculated with (2) and it is $27,900 €$. Figures 10 and 11 also represent the cost of the different setups, varying $C_{D M}$ and $C_{P R}$ in the range depicted in the first and last columns and rows of Tables 2-4. They represent the cheapest and most expensive configurations, varying with respect to the cost of $C_{D M}$ and $C_{P R}$.

Table 2. Cost $(€)$ analysis as a function of $C_{D M}$ and $C_{P R}$ Setup \#2.

\begin{tabular}{ccccccc}
\hline & & \multicolumn{5}{c}{$\boldsymbol{C}_{\boldsymbol{P R}}$} \\
\hline \multirow{6}{*}{$C_{D M}$} & $\mathbf{5 0 0}$ & $\mathbf{1 6 2 5}$ & $\mathbf{2 7 5 0}$ & $\mathbf{3 8 7 5}$ & $\mathbf{5 0 0 0}$ \\
& $\mathbf{2 0 0 0}$ & 52,100 & 54,350 & 56,600 & 58,850 & 61,100 \\
& $\mathbf{6 5 0 0}$ & 61,100 & 63,350 & 65,600 & 67,850 & 70,100 \\
& $\mathbf{1 1 , 0 0 0}$ & 70,100 & 72,350 & 74,600 & 76,850 & 79,100 \\
& $\mathbf{1 5 , 5 0 0}$ & 79,100 & 81,350 & 83,600 & 85,850 & 88,100 \\
& $\mathbf{2 0 , 0 0 0}$ & 88,100 & 90,350 & 92,600 & 94,850 & 97,100 \\
\hline
\end{tabular}

Table 3. Cost $(€)$ analysis as a function of $C_{D M}$ and $C_{P R}$-Setup \#3.

\begin{tabular}{ccccccc}
\hline & & \multicolumn{5}{c}{$\boldsymbol{C}_{\boldsymbol{P R}}$} \\
\hline \multirow{6}{*}{$C_{D M}$} & $\mathbf{5 0 0}$ & $\mathbf{1 6 2 5}$ & $\mathbf{2 7 5 0}$ & $\mathbf{3 8 7 5}$ & $\mathbf{5 0 0 0}$ \\
& $\mathbf{2 0 0 0}$ & 59,100 & 65,850 & 72,600 & 79,350 & 86,100 \\
& $\mathbf{6 5 0 0}$ & 72,600 & 79,350 & 86,100 & 92,850 & 99,600 \\
& $\mathbf{1 1 , 0 0 0}$ & 86,100 & 92,850 & 99,600 & 106,350 & 113,100 \\
& $\mathbf{1 5 , 5 0 0}$ & 99,600 & 106,350 & 113,100 & 119,850 & 126,600 \\
& $\mathbf{2 0 , 0 0 0}$ & 113,100 & 119,850 & 126,600 & 133,350 & 140,100 \\
\hline
\end{tabular}

Table 4. Cost $(€)$ analysis as a function of $C_{D M}$ and $C_{P R}$ - Setup \#4.

\begin{tabular}{ccccccc}
\hline & & \multicolumn{5}{c}{$\boldsymbol{C}_{\boldsymbol{P R}}$} \\
\hline & & $\mathbf{5 0 0}$ & $\mathbf{1 6 2 5}$ & $\mathbf{2 7 5 0}$ & $\mathbf{3 8 7 5}$ & $\mathbf{5 0 0 0}$ \\
& $\mathbf{2 0 0 0}$ & 72,100 & 78,850 & 85,600 & 92,350 & 99,100 \\
$C_{D M}$ & $\mathbf{6 5 0 0}$ & 99,100 & 105,850 & 112,600 & 119,350 & 126,100 \\
& $\mathbf{1 1 , 0 0 0}$ & 126,100 & 132,850 & 139,600 & 146,350 & 153,100 \\
& $\mathbf{1 5 , 5 0 0}$ & 153,100 & 159,850 & 166,600 & 173,350 & 180,100 \\
& $\mathbf{2 0 , 0 0 0}$ & 180,100 & 186,850 & 193,600 & 200,350 & 207,100 \\
\hline
\end{tabular}




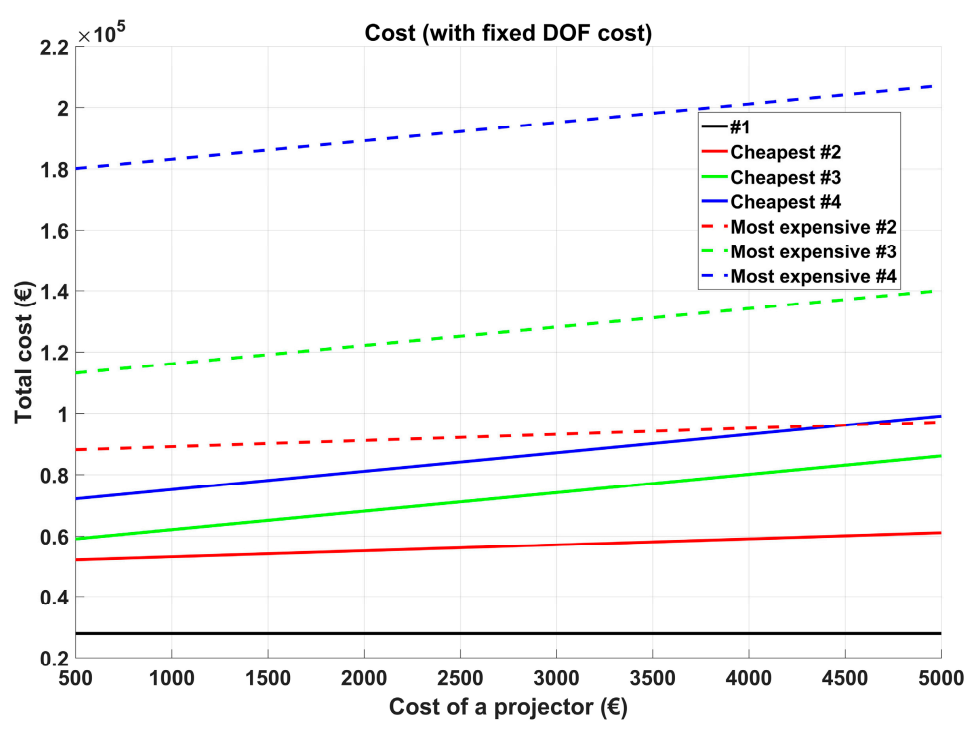

Figure 10. Cost comparison $(€)$ with fixed $C_{D M}$.

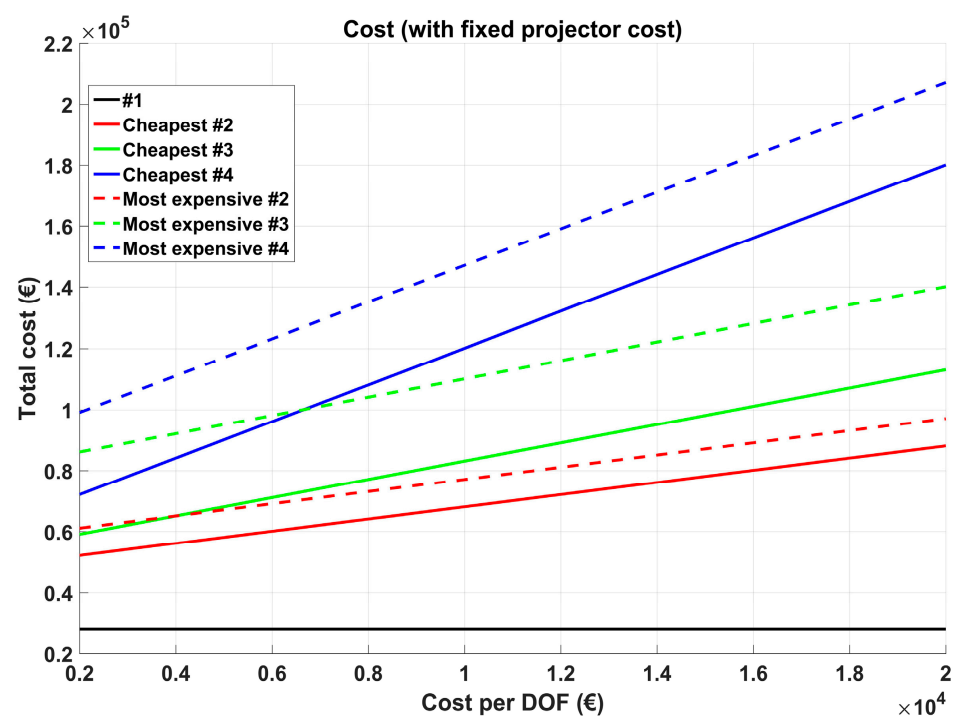

Figure 11. Cost comparison $(€)$ with fixed $C_{P R}$.

\section{Discussion}

As it is shown, the cost of setup \#1 can be considered constant, since it does not feature projectors or a motion platform and it is easier to estimate. Its cost can almost be completely attributed to the software. However, this setup is not fully suitable in terms of vehicle controls for this case study. One of the reasons is that the throttle in a speedboat is not controlled by means of a pedal, as in a car. An additional joystick can be added, but the interaction would not be natural, since a joystick is different from the speedboat throttle. The use of a TV screen offers an affordable solution, but the amount of immersion is reduced as extra-foveal visual information is lost, and consequently, the pilots do not tend to truly believe that they are experiencing and alternative reality, which is key in Virtual Reality.

Setup \#2 represents a significant improvement with respect to the first one, as it includes motion and a bigger screen. Still, extra-foveal information is lost because of the lack of a truly immersive display. Its cost can be several times higher than the first setup, especially if the motion platform is a powerful one and projectors are expensive. Another major improvement is the addition of real vehicle controls. This would be mandatory if the simulator needs to be used for skills training. 
Setup \#3 represents a further improvement with respect to the second one. The use of three screens does not raise the cost too much if the projectors are not very expensive, compared with the cost of the motion system. Yet, it provides an immersive display. The addition of the heave DOF is an important improvement that can payoff since adding DOFs is cheaper than changing from a motionless to a motion-based system. The addition of the wind generation system and a compass is relatively cheap and it would be very beneficial for the simulator.

Setup \#4 represents the most advanced setup. However, it is considerably more expensive than the third one. The use of a cylindrical screen raises the costs and the complexity of the simulator, but the visual results are better, assuming similar projectors and screen technology with respect to previous setups. On the contrary, the improvement achieved when three more DOF are added may not payoff, since it significantly raises the cost, yet the surge and sway are barely noticeable in this kind of vehicle [39]. Arguably, only the addition of yaw motion is of interest. Therefore, a 4-DOF motion system would probably be more interesting in terms of cost-effectiveness, although a proper motion-fidelity assessment needs to be performed to draw deep conclusions. For other vehicles, the situation could be different. The addition of a real boat enhances the immersion sensation, but it does not significantly change how the vehicle is controlled. In addition, it complicates the installation and transport of the simulation system and adds some complexity to the system (creating projectors occlusions, for instance) that, for some uses, could be unnecessary.

The total costs vary from 27,900 to 207,100 $€$, which is a very wide range. Most of this variation is caused by the wide range of prices and features of motion platforms. Figure 11 is probably the most interesting one, since the cost of projectors have smaller variations than motion platforms.

Taking into account that a real speedboat could cost less than 15,000 $€$, the simulator can be significantly more expensive that the actual vehicle. However, fuel, port infrastructure (harbour slip, electricity, cleaning, etc.), and some other costs, can actually make the simulator affordable. In addition, the cost of software can be heavily reduced if several instances of the simulator are implemented and deployed. Finally, yet importantly, the risk of driving a speedboat can be high, as the ocean can be a risky place, and therefore, safety reasons could foster the viability of this kind of simulator.

Several improvements and alternatives can be proposed to solve some of the issues of these setups. For instance, a common problem with the projector-screen solution is that illumination needs to be properly controlled, since daylight and low-lumen projectors can degrade the solution. An alternative is to use an HMD such as Oculus Rift, as in [42]. In addition, they provide stereoscopic vision. The problem with this solution is that an HMD does not allow one to actually see the real cockpit and the sensorized vehicle controls. In addition, fatigue or sickness usually appear sooner than with screens. This could be a major drawback. Some other multimodal devices, such as smell [43], smoke, or water spray, as in [44], can be included. However, the perceptual cues provided by these devices are not essential, so they are often dispensable.

It is also important to clarify that this discussion does not consider the particular applications of the simulator. Therefore, it is a general analysis that compares the economic implications of the proposed multimodal setups, giving insights about the possible effectiveness, advantages, and disadvantages of every option, while proposing a general scheme to achieve a successful vehicle simulation. Particular considerations for each specific use of these setups could modify these conclusions.

Regarding the effectiveness of the proposed setups, this is something that highly depends on the purpose of the simulator and the indicators/metrics chosen to measure this effectiveness. The main problem with performance indicators for simulators is that meaningful measures are hard to achieve, since subjective assessments mean that some of the questions that can be assessed are subjective, such as the experience of the user, which presents large variations among groups of users, while objective indicators of the effectiveness of a simulator greatly depend on the task and the design of the indicator itself. Just the evaluation of the motion cues, for instance, is a matter of an intense scientific debate $[45,46]$. This gives an idea of the complexity of the process. 
In addition, an array of combinations could be proposed, depending on what needs to be assessed. Some simulators are assessed without performing self-motion generation. Some others require 6-DOF systems to be qualified for regulated training [47], for instance. Others, need to use real controls. Finally, some require a certain display system or a maximum delay of the visual and motion system with respect to the vehicle inputs, etc. Therefore, a single general objective assessment process for these setups cannot be provided and particular purpose-related assessments or effectiveness studies are left for future work, since ours is a generic approach.

Similarly, the economic figures are provided as a way to compare the different solutions. Absolute figures are not as important as relative values. What truly matters is to break down and relate the different costs of a simulator and to observe the relative increase/decrease in the aggregate cost that the different setups imply.

\section{Conclusions and Future Work}

Four different multimodal interactive setups for vehicle simulation have been proposed, analysed, and discussed in terms of economic cost. This is an important issue that is sometimes overlooked in scientific research. Rough figures are provided to give insights about the economic order of magnitude necessary to design and build a vehicle simulator. Setup \#1 has an approximate cost of 27,900 $€$, setup \#2 would be in a range between 52,100 and 97,100 $€$, setup \#3 within 59,100 and 140,100 $€$, and setup \#4 would be in the 72,100-207,100 € range.

Although performance measurements should be completed to calculate objective cost-effectiveness ratios, setup \#3 seems to be the most interesting one in this regard. Setup \#4 may be too expensive, while setup \#1 is too simple and setup \#2 is much more expensive than setup \#1, yet is not highly immersive. In addition, it can be enhanced to setup \#3 with a relatively small increase of money. The study is applied to a speedboat vehicle simulator, but the proposed methodology could be used for almost any vehicle simulator.

Future work includes building these four setups in order to perform objective measures to analyse the compared benefit of each of the proposed setups, using the same task, physics, and pilots, and choosing a metric that is in line with the goals that are decided for the particular use of the simulators. The problem with these performance indicators is that meaningful measures are hard to achieve, since objective indicators of the effectiveness of a simulator are highly dependent on the task and subjective assessments present large variations among groups of users.

In addition, a 4-DOF motion system can be considered as the base of the motion cueing system. It would be interesting to compare it to the 3-DOF and 6-DOF setups. Also, the use of HMD, which has raised a lot of attention lately, can be investigated as a possible alternative for the visual system. Finally, stereoscopic clues may also be studied and included in these multimodal interactive setups.

Author Contributions: Sergio Casas provided the conception of the paper and contributed to the design and software development of the multimodal setups shown. Marcos Fernández contributed to the design, hardware development and economic estimations. José V. Riera contributed to the hardware development. All authors have contributed to the paper writing and reviews.

Conflicts of Interest: The authors declare no conflicts of interest.

\section{References}

1. Allerton, D. Principles of Flight Simulation, 1st ed.; Wiley: Chichester, UK, 2009.

2. Page, L.R. Brief history of flight simulation. In Proceedings of the SimTecT 2000, Sydney, Australia, 28 February-2 March 2000; pp. 11-17.

3. Reymond, G.; Kemeny, A. Motion cueing in the renault driving simulator. Veh. Syst. Dyn. 2000, 34, $249-259$. [CrossRef]

4. Casas, S.; Olanda, R.; Dey, N. Motion cueing algorithms: A review-Algorithms, evaluation and tuning. Int. J. Virtual Augment. Real. 2017, 1, 90-106. [CrossRef]

5. Stewart, D. A platform with six degrees of freedom. Proc. Inst. Mech. Eng. 1965, 180, 371-386. [CrossRef] 
6. Hosman, R.; Advani, S. Design and evaluation of the objective motion cueing test and criterion. Aeronaut. J. 2016, 120, 873-891. [CrossRef]

7. Kemeny, A.; Panerai, F. Evaluating perception in driving simulation experiments. Trends Cogn. Sci. 2003, 7, 31-37. [CrossRef]

8. De Winter, J.; Van Leuween, P.; Happee, P. Advantages and disadvantages of driving simulators: A discussion. In Proceedings of the Measuring Behavior, Utrecht, The Netherlands, 28-31 August 2012.

9. Mauro, S.; Gastaldi, L.; Pastorelli, S.; Sorli, M. Dynamic flight simulation with a 3 dof parallel platform. Int. J. Appl. Eng. Res. 2016, 11, 9436-9442.

10. Yavrucuk, I.; Kubali, E.; Tarimci, O. A low cost flight simulator using virtual reality tools. IEEE Aerosp. Electron. Syst. Mag. 2011, 26, 10-14. [CrossRef]

11. Advani, S.; Hosman, R.; Haeck, N. Integrated design of a motion cueing algorithm and motion-base mechanism for a wright flyer simulator. In Proceedings of the AIAA Modeling and Simulation Technologies Conference, Monterey, CA, USA, 5-8 August 2002.

12. Reid, L.D.; Nahon, M.A. Response of airline pilots to variations in flight simulator motion algorithms. J. Aircr. 1988, 25, 639-646. [CrossRef]

13. Wei, F.-S.; Amaya-Bower, L.; Gates, A.; Rose, D.; Vasko, T. The full-scale helicopter flight simulator design and fabrication at CCSU. In Proceedings of the 57th AIAA/ASCE/AHS/ASC Structures, Structural Dynamics, and Materials Conference, San Diego, CA, USA, 4-8 January 2016; p. 0582.

14. Wiskemann, C.M.; Drop, F.M.; Pool, D.M.; Van Paassen, M.M.; Mulder, M.; Bülthoff, H.H. Subjective and objective metrics for the evaluation of motion cueing fidelity for a roll-lateral reposition maneuver. In Proceedings of the American Helicopter Society 70th Annual Forum, Montreal, QC, Canada, 20-22 May 2014.

15. Schroeder, J.A. Helicopter Flight Simulation Motion Platform Requirements; National Aeronautics and Space Administration: Moffett Field, CA, USA, 1999.

16. Jansson, J.; Sandin, J.; Augusto, B.; Fischer, M.; Blissing, B.; Källgren, L. Design and performance of the VTI sim IV. In Proceedings of the Driving Simulation Conference, Paris, France, 4-5 September 2014.

17. Nehaoua, L.; Mohellebi, H.; Amouri, A.; Arioui, H.; Espié, S.; Kheddar, A. Design and control of a small-clearance driving simulator. IEEE Trans. Veh. Technol. 2008, 57, 736-746. [CrossRef]

18. Chapron, T.; Colinot, J.P. The new psa peugeot-citroën advanced driving simulator overall design and motion cue algorithm. In Proceedings of the Driving Simulation Conference, Iowa City, IA, USA; 2007.

19. Avizzano, C.A.; Barbagli, F.; Bergamasco, M. Washout filter design for a motorcycle simulator. In Proceedings of the IEEE Conference on Systems, Man, and Cybernetics, Nashville, TN, USA, 8-11 October 2000; IEEE: Nashville, TN, USA; pp. 995-1000.

20. Nehaoua, L.; Hima, S.; Arioui, H.; Seguy, N.; Espié, S. Design and modeling of a new motorcycle riding simulator. In Proceedings of the American Control Conference, New York, NY, USA, 9-13 July 2007; IEEE: Nashville, TN, USA; pp. 176-181.

21. Cossalter, V.; Lot, R.; Massaro, M.; Sartori, R. Development and validation of an advanced motorcycle riding simulator. Proc. Inst. Mech. Eng. D J. Automob. Eng. 2011, 225, 705-720. [CrossRef]

22. Thöndel, E. Design and optimisation of a motion cueing algorithm for a truck simulator. In Proceedings of the European Simulation and Modelling Conference-ESM, Essen, Germany, 22-24 October 2012.

23. Lleras, N.O.; Brennan, S.; Murphy, D.; Klena, M.J.; Garvey, P.M.; Sommer, H., III. Development of an open-source tractor driving simulator for tractor stability tests. J. Agric. Saf. Health 2016, 22, 227-246.

24. Filipczuk, P.; Nikiel, S. Real-time simulation of a sailboat. In Proceedings of the 2008 Conference on Human System Interactions, Krakow, Poland, 25-27 May 2008; IEEE: Nashville, TN, USA; pp. 970-974.

25. Casas, S.; Rueda, S.; Riera, J.V.; Fernández, M. On the real-time physics simulation of a speed-boat motion. In Proceedings of the GRAPP/IVAPP, Rome, Italy, 24-26 February 2012; pp. 121-128.

26. Abásolo, M.J.; Bauza, C.G.; Lazo, M.; D’Amato, J.P.; Vénere, M.; De Giusti, A.; Manresa-Yee, C.; Mas-Sansó, R. From a serious training simulator for ship maneuvering to an entertainment simulator. In Proceedings of the International Conference on Articulated Motion and Deformable Objects, Palma de Mallorca, Spain, 16-18 July 2014; Springer: Berlin, Germany; pp. 106-117.

27. Ueng, S.-K.; Lin, D.; Liu, C.-H. A ship motion simulation system. Virtual Real. 2008, 12, 65-76. [CrossRef]

28. Xiufeng, Z.; Yicheng, J.; Yong, Y.; Zhihua, L. Ship simulation using virtual reality technique. In Proceedings of the 2004 ACM SIGGRAPH International Conference on Virtual Reality Continuum and its Applications in Industry, Singapore, 16-18 June 2004; ACM: New York, NY, USA; pp. 282-285. 
29. Schulzyk, O.; Hartmann, U.; Bongartz, J.; Bildhauer, T.; Herpers, R. A real bicycle simulator in a virtual reality environment: The fivis project. In Proceedings of the 4th European Conference of the International Federation for Medical and Biological Engineering, Antwerp, Belgium, 23-27 November 2008; Springer: Berlin, Germany; pp. 2628-2631.

30. Herpers, R.; Heiden, W.; Kutz, M.; Scherfgen, D.; Hartmann, U.; Bongartz, J.; Schulzyk, O. Fivis bicycle simulator: An immersive game platform for physical activities. In Proceedings of the 2008 Conference on Future Play: Research, Play, Share, Toronto, ON, Canada, 3-5 November 2008; ACM: New York, NY, USA; pp. 244-247.

31. Allison, R.S.; Harris, L.R.; Hogue, A.; Jasiobedzka, U.; Jenkin, H.; Jenkin, M.; Jaekl, P.; Laurence, J.; Pentile, G.; Redlick, F. Simulating self-motion ii: A virtual reality tricycle. Virtual Real. 2002, 6, 86-95. [CrossRef]

32. Mullen, N.; Charlton, J.; Devlin, A.; Bedard, M. Simulator Validity: Behaviors Observed on the Simulator and on the Road; CRC Press: Boca Raton, FL, USA, 2011.

33. Kaptein, N.; Theeuwes, J.; Van Der Horst, R. Driving simulator validity: Some considerations. Transp. Res. Rec. 1996, 1550, 30-36. [CrossRef]

34. Grant, P.R.; Reid, L.D. Motion washout filter tuning: Rules and requirements. J. Aircr. 1997, 34, $145-151$. [CrossRef]

35. Zhao, J.; Yun, M.; Zhang, H.M.; Yang, X. Driving simulator evaluation of drivers' response to intersections with dynamic use of exit-lanes for left-turn. Accid. Anal. Prev. 2015, 81, 107-119. [CrossRef] [PubMed]

36. Arioui, H.; Hima, S.; Nehaoua, L. 2 dof low cost platform for driving simulator: Modeling and control. In Proceedings of the IEEE/ASME International Conference on Advanced Intelligent Mechatronics, Singapore, 14-17 July 2009; pp. 1206-1211.

37. Huang, A.R.W.; Chen, C. A low-cost driving simulator for full vehicle dynamics simulation. IEEE Trans. Veh. Technol. 2003, 52, 162-172. [CrossRef]

38. Nahon, M.A.; Reid, L.D. Simulator motion-drive algorithms-A designer's perspective. J. Guid. Control Dyn. 1990, 13, 356-362. [CrossRef]

39. Casas, S.; Coma, I.; Riera, J.V.; Fernandez, M. On the characterization of a speed-boat motion for real-time motion cueing. In Proceedings of the GRAPP/IVAPP, Barcelona, Spain, 21-24 February 2013; pp. 275-278.

40. Bertollini, G.; Glase, Y.; Szczerba, J.; Wagner, R. The effect of motion cueing on simulator comfort, perceived realism, and driver performance during low speed turning. In Proceedings of the Driving Simulator Conference, Paris, France, 4-5 September 2014.

41. Colombet, F.; Dagdelen, M.; Reymond, G.; Pere, C.; Merienne, F.; Kemeny, A. Motion cueing: What's the impact on the driver's behaviour? In Proceedings of the Driving Simulator Conference, Monte-Carlo, Monaco, 31 January-1 February 2008; pp. 171-182.

42. Jose, R.; Lee, G.A.; Billinghurst, M. A comparative study of simulated augmented reality displays for vehicle navigation. In Proceedings of the 28th Australian Conference on Computer-Human Interaction, Launceston, Australia, 29 November-2 December 2016; ACM: New York, NY, USA; pp. 40-48.

43. Olorama. Available online: http://www.olorama.com/en/ (accessed on 1 March 2017).

44. Casas, S.; Portalés, C.; García-Pereira, I.; Fernández, M. On a first evaluation of romot—A robotic 3d movie theatre-for driving safety awareness. Multimodal Technol. Interact. 2017, 1, 6. [CrossRef]

45. Hosman, R. Are criteria for motion cueing and time delays possible? In Proceedings of the AIAA Modeling and Simulation Technologies Conference, Portland, OR, USA, 9-11 August 1999.

46. Hosman, R.; Advani, S. Are criteria for motion cueing and time delays possible? Part 2. In Proceedings of the AIAA Modeling and Simulation Technologies Conference, Boston, MA, USA, 19-22 August 2013.

47. DNV. Standard for Certification Maritime Simulator Systems No. 2.14. Available online: https://rules.dnvgl. com/docs/pdf/DNV/stdcert/2011-01/Standard2-14.pdf (accessed on 1 March 2017).

(C) 2017 by the authors. Licensee MDPI, Basel, Switzerland. This article is an open access article distributed under the terms and conditions of the Creative Commons Attribution (CC BY) license (http://creativecommons.org/licenses/by/4.0/). 